\title{
Publisher's Note: High-resolution hard x-ray magnetic imaging with dichroic ptychography [Phys. Rev. B 94, 064421 (2016)]
}

Claire Donnelly, Valerio Scagnoli, Manuel Guizar-Sicairos, Mirko Holler, Fabrice Wilhelm, Francois Guillou, Andrei Rogalev, Carsten Detlefs, Andreas Menzel, Jörg Raabe, and Laura J. Heyderman

(Received 1 November 2016; published 10 November 2016)

DOI: 10.1103/PhysRevB.94.179903

This paper was published online on 17 August 2016 with an error in Eq. (4) and the following text. Equation (4) should read as

$$
\mathbf{O}\left(\mathbf{r}-\mathbf{r}_{j}\right)=\exp \left\{i \frac{\omega}{c} \int\left[1-\frac{r_{e}}{2 \pi} \lambda^{2} n_{\text {at }} f\left(\mathbf{r}-\mathbf{r}_{j}\right)\right] d z\right\}
$$

The text following Eq. (4) should read as " $\Psi_{j}(\mathbf{r}), \mathbf{P}_{j}(\mathbf{r})$, and $\mathbf{O}\left(\mathbf{r}-\mathbf{r}_{j}\right)$ are all complex-valued functions of $\mathbf{r}$ which are the transverse Cartesian coordinates, and $j$ indicates the ptychography scan position. Here $\omega$ is the frequency of the $\mathrm{x}$ rays, $c$ is the speed of light, $r_{e}$ is the classical electron radius, $\lambda$ is the wavelength of the $\mathrm{x}$ rays, $n_{\mathrm{at}}$ is the atomic density of a particular element, and $f\left(\mathbf{r}-\mathbf{r}_{j}\right)$ is the scattering factor [defined in Eq. (1)]." The equation and text have been corrected as of 26 October 2016 . The equation and text are incorrect in the printed version of the journal. 